Catherine Van Nieuwenhoven

Université catholique de Louvain

Nicolas Roland

Université Libre de Bruxelles

\title{
La formation pratique des enseignants en Belgique francophone
}

\section{Chronique internationale}

\section{Introduction}

Cette contribution vise à dresserun état des lieux de la formation pratique des enseignants de l'enseignement fondamental et secondaire en Belgique et, plus particulièrement, en Fédération Wallonie-Bruxelles (FWB) - la partie francophone du pays -, anciennement dénommée Communautéfrançaise de Belgique (CFB). Le cursus scolaire d'un élève en Belgique se découpe, quant à lui, en quatre grandes périodes: l'enseignement maternel de 2 ans et demi à 6 ans (non obligatoire, mais fortement recommandé), l'enseignement primaire de 6 ans à 12 ans (obligatoire), l'enseignement secondaire de 12 ans à 18 ans (obligatoire) et, enfin, l'enseignement supérieur. À l'heure actuelle, la formation des enseignants des sections préscolaire (3-5 ans), primaire (6-12 ans) et secondaire inférieur (12-15 ans) est organisée en trois années au sein de hautes écoles (établissements d'enseignement supérieur de type court) et donne accès à un « bachelier » en trois ans; celle des enseignants du secondaire supérieur (16-18 ans) est organisée en 5 années, voire en 6 années à l'université (établissement d'enseignement supérieur de type long) et donne accès à un «Master ». Dans le présent article, nous décrivons les différentes modalités de la formation pratique des futurs enseignants tant en haute école qu'à l'université en nous basant sur deux études de cas. Nous terminons en soulevant les questions ainsi que les défis de cette formation pratique et éclairons le lecteur sur les évolutions futures de la formation des enseignants. 


\section{CHROMLOLIE}

\section{La formation pratique des futurs enseignants en haute école}

\section{Introduction}

En 2001, la réforme de la formation initiale des enseignants en Belgique francophone a induit divers changements institutionnels et organisationnels importants: imposition d'un référentiel de 13 compétences $^{1}$, d'un nouveau cahier des charges avec des intitulés de cours actualisés et d'une réorganisation des stages. Cette réforme s'est appuyée sur le décret "Missions » (1997) qui énonce pour tous une approche par compétences. À ce sujet, Frenay et Maroy (2004) ont écrit que ce décret (1997) s'avère le tremplin à d'autres textes légaux qui s'y réfèreront à visée d'approfondissement.

\section{La réforme du paysage du supérieur}

Ce sera ainsi le cas également du décret (FWB, 7 novembre 2013) définissant le paysage de l'enseignement supérieur et l'organisation académique des études édicté par le ministre Marcourt. Ce décret vise à centrer la formation sur le parcours de l'étudiant et à garantir la cohérence de l'enseignement supérieur autour de la création de l'ARES (Académie de recherche et d'enseignement supérieur), comme seule instance de pilotage.

Selon l'article 121, ce sont les autorités académiques de l'établissement d'enseignement supérieur qui établissent les profils d'enseignement, les programmes et les calendriers détaillés des activités d'apprentissage, regroupées en unités d'enseignement (UE), des études pour lesquelles leur établissement est habilité en tenant compte du programme minimal établi et du référentiel de compétences (FWB, 7 novembre 2013). Ce sont les acquis d'apprentissage terminaux (AAT) qui déterminent les résultats attendus en fin de formation. Ils sont définis sur base du référentiel de compétences officiel ${ }^{2}$ et du profil d'enseignement propre à chaque institution. Les AAT sont ensuite déclinés en acquis d'apprentissages intermédiaires (AAI) et en acquis d'apprentissage spécifiques (AAS) pour chacune des unités d'enseignement qui regroupent les activités d'apprentissage qui poursuivent un même objectif. La figure 1 modélise les balises qui fondent le programme de la formation des enseignants.

\section{La formation pratique par les stages et les ateliers de formation professionnelle}

La formation des enseignants se veut davantage " professionnalisante » et a pour but de former un enseignant développant une identité professionnelle forte et capable de s'adapter aux nouveaux défis de transformation de l'école. Le temps accordé aux stages y est important, couvrant près de 500 heures. Les stages sont, selon le décret de 2000, «des activités pédagogiques pratiques en situation réelle » (CFB, 2000, article 15, p. 5). Ces stages, fondements de la formation initiale des enseignants, sont des lieux privilégiés d'expérimentations, de réflexion, d'engagement dans un métier collectif, d'affirmation du soi professionnel et de développement personnel.

1 Ministère de la CFB, décret du 12 décembre 2000, p. 2.

2 Ce référentiel s'articule maintenant autour de sept compétences qui s'inscrivent dans le cadre européen des certifications (CEC). 
En haute école, la rencontre avec la pratique est organisée de manière progressive par des stages d'observation et se poursuit par des stages en responsabilité dès la deuxième année, avec des durées de plus en plus longues. Ainsi le décret du 12 décembre 2000 impose deux semaines de stage en première année, quatre semaines en deuxième année et dix semaines en troisième année, et ce, pour toutes les sections. Alors qu'en première année, l'étudiant est surtout amené à observer le fonctionnement d'une classe, la gestion des apprentissages et à planifier des séquences d'apprentissage en prenant en charge l'une ou l'autre activités, lors de la deuxième année, une alternance de type intégrative est mise en place et conduit l'étudiant à passer à plusieurs reprises de l'institut de formation à plusieurs lieux de pratique. L'alternance n'est pas formatrice en tant que telle, elle n'est que la condition nécessaire d'une articulation théorie-pratique.

C'est dans ce cadre que le législateur a introduit, dès 2001, un dispositif innovant, appelé les ateliers de formation professionnelle (AFP) pour tisser des liens entre les deux lieux de formation. Leur objectif est de placer théorie et pratique dans un rapport de fonctionnalité réciproque : la théorie sert à élaborer et réguler les processus d'enseignement alors que la pratique sert à contextualiser, éprouver et réorganiser les contenus théoriques, dans le contexte du développement des compétences professionnelles. Ces ateliers sont coanimés par des didacticiens, des psychopédagogues et des nouveaux partenaires, à savoir des maitres de formation pratique (MFP) présents pour témoigner de leur expertise du terrain. Au sein de la réforme de 2013, ces AFP sont placés en articulation directe avec les stages et intégrés dans les mêmes unités d'enseignement.

Selon le texte officiel, l'objectif central des AFP est «d'expérimenter, d'observer et d'analyser les différentes composantes de la profession. Ils intègrent la didactique disciplinaire et générale dans le cadre d'activités organisées à la fois sur le terrain et à la haute école »(CFB, 2000, Article 10, p. 4). Concrètement, même si les AFP constituent un lieu privilégié d'intégration, ils ne suffisent pas à développer chez l'étudiant la posture réflexive nécessaire pour répondre à la complexité des tâches et des situations professionnelles (Perrenoud, 2001). C'est pourquoi les dispositifs qui soutiennent les stages ont également un rôle essentiel dans l'accompagnement du développement d'une posture réflexive chez l'étudiant pour le rendre capable d'adapter ses actions aux contextes mouvants et difficiles qu'il rencontre sur le terrain (Nitonde et Paquay, 2011). Ensemble, les AFP et les stages couvrent près d'un tiers de la formation. Pour aborder l'accompagnement et l'évaluation des stages, nous nous référons au dispositif mis en place par l'Institut supérieur de pédagogie Galilée (ISPG) afin d'illustrer la traduction du prescrit sur le terrain.

\section{L'accompagnement des étudiants en stage}

L'accompagnement des étudiants en stage est dévolu, dans l'institut de formation, aux superviseurs (psychopédagogues et didacticiens) ainsi que, sur le terrain, aux maitres de stage. Très rapidement est apparue la nécessité de développer le pôle formatif de l'accompagnement des stages. Ainsi, l'encadrement des stages par l'institut de formation est assuré par deux interlocuteurs différents pour chaque étudiant : le superviseur qui assure l'accompagnement formatif, dans sa dimension relationnelle, méthodologique et institutionnelle, des étudiants, tout au long d'une année académique (soit en $2^{\mathrm{e}}$, soit en $3^{\mathrm{e}}$ ) et le professeur visiteur qui atteste de la maitrise des compétences visées par chaque stage. Il est entendu qu'un même professeur peut assurer les deux rôles simultanément pour un stage, mais pas pour les mêmes étudiants. Il est même souhaitable que les superviseurs d'une année aient une charge 
de cours auprès des étudiants qu'ils encadrent. Pour préparer leur stage, les étudiants disposent d'un temps d'observation de leur classe et d'une semaine de préparation de leurs séquences didactiques avec des aller-retour entre leurs enseignants et le maitre de stage. Des temps forts jalonnent aussi le dispositif d'accompagnement de chaque étudiant en stage en deuxième et troisième année :

- une rencontre collective avec le superviseur et les autres étudiants qui gravitent autour de celuici pour inscrire l'accompagnement dans une dynamique réflexive collective et prendre sa propre pratique comme objet de réflexion, voire de théorisation;

- $\quad$ un entretien individuel avant le début du stage pour fixer le contrat de stage et identifier les objectifs personnalisés poursuivis par l'étudiant;

- un entretien « à chaud » sur place à la suite de l'observation d'une séquence de cours en présence du maitre de stage. Cet échange en triade permet d'amener l'étudiant, par un questionnement orienté, à une prise de conscience de son fonctionnement et à identifier des pistes de régulation à court terme;

- un entretien réflexif individuel avec le superviseur comprenant une étape de description objective d'une situation de stage, une étape d'analyse de la situation (aider l'étudiant à expliciter ce qu'il perçoit qui a bien ou moins bien fonctionné), une interprétation de la situation (explicitation des choix et comprendre les effets des actions menées) et une étape créative de mise en projet (dégager les priorités pour l'activité d'enseignement future en précisant les critères d'observation-évaluation).

La circulaire du 14 juin 2001 (FWB) précise que le maitre de stage joue aussi un rôle formatif dans l'accompagnement de l'étudiant en l'aidant à dépasser le cadre de la reproduction au profit d'une pratique professionnelle réflexive. Toutefois, Van Nieuwenhoven et Colognesi (2013) signalent que le décret prévoit, pour ces accompagnateurs, que les instituts de formation puissent leur assurer une formation complémentaire, mais dans les faits, aucune formation ne leur est actuellement proposée. Ils ne sont dès lors pas préparés à ce rôle d'accompagnateurs de jeunes débutants.

Globalement, pour assurer une cohérence entre les objectifs de stage poursuivis par l'institut de formation et ceux que les maitres de stage décodent et acceptent de poursuivre en fonction de leur contexte professionnel, un minimum de culture commune des formateurs-accompagnateurs est nécessaire.

\section{L'évaluation des stages}

Le conseil d'évaluation est la dernière étape du dispositif d'accompagnement des stages et met en présence le stagiaire, le superviseur, le professeur visiteur et le maitre de stage s'il a pu se libérer (dans le cas contraire, son rapport est commenté par le superviseur). Le superviseur initie le conseil et en assume la conduite. C'est donc collégialement que la note de l'étudiant est établie sur base de l'autoévaluation de l'étudiant, des rapports du superviseur et du maitre de stage en tant que témoins du parcours et de la progression de l'étudiant et du rapport du visiteur qui atteste de la maitrise des compétences visées lors de sa visite, sur base d'appréciations et de commentaires rédigés pour argumenter sa position et alimenter la discussion. Les quatre acteurs se basent sur une même grille d'évaluation, construite sur base du référentiel des 7 compétences et ventilée en critères de maitrise. Un seuil minimal de réussite 
est identifié pour chacune des compétences et fonctions des stages. Au terme du conseil, l'étudiant reçoit un avis global et indicatif (réussite, balance, échec). La note finale est établie pour chacun des étudiants par l'ensemble des superviseurs, en fin d'année, sur base des bilans des deux stages réalisés.

Des tensions peuvent exister entre la dynamique formative liée à la régulation des apprentissages de l'étudiant et à celle qui s'inscrit dans une visée certificative nécessaire à l'établissement d'un profil de compétences de l'étudiant en fin de parcours. C'est pourquoi l'ISPG a décidé de distinguer ces deux fonctions de l'évaluation et de favoriser l'accompagnement de la progression de l'étudiant. C'est dans cette perspective de soutien accru à la réflexivité (Campanale, 1997; Saussez et Allal, 2007) que l'autoévaluation de l'étudiant est suscitée tout au long des stages par l'intermédiaire d'un carnet de bord, de grilles d'observation critériées. Ces outils réflexifs sont réinvestis lors des rencontres de supervision collective et individuelle.

\section{La formation pratique des futurs enseignants à l'université}

\section{Introduction}

À l'université, deux voies existent pour devenir enseignant dans le secondaire supérieur : d'une part, effectuer un baccalauréat ( 3 ans) suivi d'un master disciplinaire ( 2 ans) et, ensuite, d'une année supplémentaire pour obtenir " l'agrégation de l'enseignement secondaire supérieur », correspondant à un programme de formation de 30 crédits (ou ECTS). Un baccalauréat (3 ans) à l'université, suivi d'un master à finalité didactique (2 ans) correspondant à deux années d'études et 120 crédits (ou ECTS), dont 30 spécifiques de formation pédagogique (Denis, 2013). En effet, depuis $2004^{3}$, les études de deuxième cycle universitaire ("master») sont organisées selon trois finalités différentes : premièrement, la finalité dite « spécialisée », préparant à l'exercice d'une ou de plusieurs professions liées à un champ disciplinaire particulier; deuxièmement, la finalité dite «didactique », préparant à l'enseignement de cette (ou de ces) discipline(s); troisièmement, la finalité dite «approfondie ", préparant plus particulièrement à la recherche dans le domaine considéré.

De manière commune à l'ensemble des établissements, la formation pédagogique des enseignants à l'université correspond à 300 heures de cours et de stages. Toutefois, les universités disposent de 30 \% de marge de manœuvre pour adapter leur curriculum (Mathy, 2011). Cette formation comprend quatre axes : les connaissances socioculturelles (30 heures ou plus), les connaissances pédagogiques assorties d'une démarche scientifique et d'attitudes de recherche (60 heures ou plus), les connaissances socioaffectives et relationnelles (30 heures ou plus) et, enfin, le savoir-faire ( 90 heures ou plus).

\section{L'axe savoir-faire : préparation, action et réflexion}

Le temps consacré à la formation pratique des enseignants - l'axe «savoir-faire » - diverge en fonction des établissements, mais des éléments communs se dégagent : premièrement, des cours ou exercices préalables aux stages tels que le microenseignement (autoscopie) sont proposés aux étudiants. Ces séances formatives d'enseignement filmé centrées sur la mise en œuvre d'habiletés pédagogiques

3 Décret de la Communauté française de Belgique du 31-03-04. 
ciblées permettent aux étudiants de se préparer à la phase d'enseignement devant une classe réelle. Deuxièmement, l'axe savoir-faire offre trois types de stages: des stages d'observation, des stages d'enseignement ainsi que des stages d'implication « hors cours ", c'est-à-dire proposant une implication dans des activités liées au fonctionnement de l'établissement et aux relations entre ses acteurs. Enfin, des séances de pratique réflexive permettent aux futurs enseignants de prendre du recul par rapport à leurs pratiques, leurs représentations et de réfléchir sur leurs manières d'agir (Paquay, 2005).

\section{La formation pratique par les stages}

La formation pratique des enseignants par les stages à l'université est peu documentée, tant dans les textes officiels que dans les programmes de cours des institutions universitaires. Légalement, l'étudiant doit effectuer un minimum de 60 heures effectives de stage (qui comprend l'observation préalable des classes, les préparations et les activités pédagogiques hors leçons). Qui plus est, une diversification des lieux de stage doit permettre aux étudiants de rencontrer le plus de situations professionnelles possible - allant de l'enseignement général à l'enseignement professionnel en passant, parfois, par l'enseignement spécialisé (Mathy, 2011). Ces stages actifs en milieu réel d'enseignement sont supervisés par le professeur de didactique de la discipline ainsi que par un maitre de stage dans chaque établissement. Le superviseur conseille l'étudiant en amont de sa pratique de stage et encadre sa réalisation. Il assiste également à au moins deux leçons appelées « Leçons publiques d'examen ».

Parmi les programmes de cours disponibles sur Internet, celui de l'Agrégation de l'enseignement secondaire supérieur «Information et communication » de l'Université catholique de Louvain (UCL) ${ }^{4}$ présente de manière précise les différentes modalités organisationnelles et pédagogiques liées aux stages; nous utilisons ce programme en guise d'illustration pour offrir au lecteur une description des activités de la formation pratique par les stages à l'université.

\section{Le contenu des stages}

Dans ce programme, les stages se découpent en trois parties : d'une part, plusieurs stages d'observation et d'analyse de pratiques pédagogiques préalables à l'enseignement d'une durée de 10 heures avec un minimum de 2 heures par classe; d'autre part, trois stages d'enseignement, dont deux d'une durée de 16 heures et un d'une durée de 8 heures. Ces derniers comprennent trois phases distinctes: l'observation des classes et la préparation des leçons (avant), les leçons prestées en classe (pendant) et l'évaluation formative des leçons (après). Enfin, la dernière partie concerne la participation à une activité pédagogique d'une durée de 10 heures.

\section{L'évaluation des stages}

Au sein de l'Agrégation de l'enseignement secondaire supérieur «Information et communication » de l'UCL, les stages sont évalués sur base d'une grille critériée remise aux étudiants dès le début de l'année académique. Cette grille est utilisée tant par l'étudiant dans le cadre de son autoévaluation - afin de favoriser sa pratique réflexive - que par l'ensemble des évaluateurs - superviseur et maitre de stage.

La cote finale attribuée pour les stages est pondérée en fonction de 4 dimensions : les informations contenues dans la farde de stage de l'étudiant-stagiaire, les prestations des étudiants-stagiaires lors

4 http://www.uclouvain.be/cours-2014-LAGES9009 
des leçons publiques d'examen en présence des superviseurs et l'évolution de ces prestations, le rapport commenté des maitres de stage ainsi que le rapport de stage de l'étudiant-stagiaire (capacité d'autoévaluation). En outre, les leçons publiques d'examen constituent un moment clé parmi les sources de l'évaluation; elles possèdent donc une pondération particulière dans l'attribution de la note finale.

\section{Conclusion}

En guise de conclusion, nous proposons au lecteur quelques enjeux liés à la formation pratique des enseignants en Fédération Wallonie-Bruxelles avant de décrire les évolutions futures de la formation des enseignants.

Premièrement, à l'heure actuelle, il existe peu de balises structurant les rôles attendus des maitres de stage, en haute école comme à l'université. Ceci engendre une impossibilité de cohérence et d'harmonisation des pratiques d'accompagnement et d'évaluation avec les acteurs de l'institut de formation. Deuxièmement, la collaboration interprofessionnelle (Dezutter, 2009) inhérente aux unités d'enseignement (regroupant des psychopédagogues, des didacticiens et des MFP) et aux stages (mettant en jeu des accompagnateurs issus de l'institution de formation et du terrain) est encore à construire. Elle exige que l'identité professionnelle de chaque groupe d'acteurs soit reconnue, mais surtout valorisée et respectée. Troisièmement, l'évaluation formative assurée durant toute la durée des stages par les accompagnateurs entre parfois en tension avec la dimension certificative exigée par l'institut de formation pour valider la maitrise des compétences chez l'étudiant. Quatrièmement, l'insertion des jeunes enseignants dans le métier reste préoccupante avec un taux de sortie élevé surtout dans l'enseignement secondaire. En effet, $35 \%$ des enseignants débutants quittent le métier endéans les cinq premières années dont $19 \%$ partent déjà durant la première année ou au terme de celle-ci (Delvaux, Desmarez, Dupriez, Lothaire et Veinstein, 2013). Enfin, nous soulignons l'importante différence en termes de temps consacré à la formation pratique des futurs enseignants entre les étudiants-stagiaires de haute école qui disposent, en moyenne, de 480 heures de "savoir-faire " alors que les étudiantsstagiaires à l'université n'ont que 90 heures consacrées à ce « savoir-faire ».

Plusieurs facteurs tels que le nombre très élevé d'élèves en difficulté à l'école, l'hétérogénéité des classes, le manque de disponibilité des élèves à apprendre ainsi que la difficulté à attirer un public motivé à s'engager et à persévérer dans le métier suscitent des questionnements sur la formation initiale des enseignants. Dans ce contexte d'accroissement des exigences envers les enseignants et d'incapacité du système éducatif à soutenir la réussite de tous les élèves, la formation des enseignants est remise en question. Dès lors, un groupe de réflexion commandité par l'ARES ${ }^{5}$ soutient qu'il faudrait avancer vers une formation de tous les enseignants correspondant au niveau $7 \mathrm{du}$ cadre européen de certification sans préjuger par contre de l'organisation des études en termes d'opérateur de référence (haute école ou université ou co-gestion), de durée, etc. La formation est un processus de longue haleine qui se déploie tout au long de la carrière, notamment par la formation continuée et le travail collaboratif suscité au sein des établissements. Ainsi, les stages resteraient la composante majeure de la formation, en articulation étroite avec les autres axes de formation - disciplinaire, didactique, pédagogique, etc. - et soutenus par une démarche réflexive auprès des étudiants. Deux autres priorités sont encore pointées par ce groupe : une formation en sciences sociales qui pourrait offrir un cadre d'analyse pour soutenir le recul réflexif

5 Groupe de travail dit des « quatre opérateurs » de la formation initiale (GT4O). 
des étudiants sur les situations professionnelles rencontrées et une formation réellement adossée à la recherche en éducation, entre autres par la réalisation du travail de fin d'études ou mémoire, mais aussi par l'implication dans des recherches.

\section{Références}

Campanale, F. (1997). Autoévaluation et transformation de pratiques pédagogiques. Mesure et évaluation en éducation, 20(1), 1-24. Repéré à https://hal.archives-ouvertes.fr/hal-01111189

Décret définissant la formation initiale des instituteurs et régents : Décret de la Communauté française du 12 décembre 2000 définissant la formation initiale des instituteurs et régents. (2001). Moniteur belge, 19 janvier 2001. Repéré à http://www.gallilex.cfwb.be/document/pdf/25501 000.pdf

Décret définissant les missions prioritaires de l'enseignement fondamental et de l'enseignement secondaire et organisant les structures propres à les atteindre : Décret de la Communauté française du 24 juillet 1997. Moniteur belge, 23 septembre 1997. Repéré à http://www.gallilex.cfwb.be/fr/leg res 01.php?ncda=21557\&referant=101

Décret du 07.11.2013 définissant le paysage de l'enseignement supérieur et l'organisation académique des études. Repéré à http://www.gallilex.cfwb.be/document/pdf/39681 005.pdf

Delvaux, B., Desmarez, P., Dupriez, V., Lothaire, S. et Veinstein, M. (2013). Les enseignants débutants en Belgique francophone : trajectoires, conditions d'emploi et positions sur le marché du travail. Les cabiers de recherche $d u$ GIRSEF, 92.

Dezutter, O. (2009). La collaboration interprofessionnelle, une clé de la continuité des apprentissages. Dans M. Coupremanne (dir.), Les dynamiques des apprentissages, la continuité au cœur des apprentissages (p. 22-34). Bruxelles : De Boeck.

Frenay, M. et Maroy, C. (2004). Lécole, six ans après le décret « Missions» : regards interdisciplinaires sur les politiques scolaires en Communauté française de Belgique. Louvain-la-Neuve : Presses universitaires de Louvain.

Mathy, J. (2011). Quelle formation initiale pour nos enseignants? Les analyses de la FAPEO. Repéré à http://www.fapeo.be/wp-content/analyses/analyses 2011/formationinitiale.pdf

Nitonde, F. et Paquay, L. (2011). Vers quelles pratiques de stage en formation initiale des enseignants du secondaire? Analyse des conceptions des enseignants de l'ENS au Burundi. Education E Formation. 295, 143-164. Repéré à http://ute3.umh.ac.be/revues/include/download.php?idRevue=11\&idRes=101

Paquay, L. (2005). Quelle formation des enseignants en Belgique francophone? Formation et profession, 11(1), 28-34. Repéré à http://www.crifpe.ca/download/verify/227

Perrenoud, P. (2001). Articulation théorie-pratique et formation de praticiens réflexifs en alternance. Dans P. Lhez, D. Millet et B. Séguier (dir.), Alternance et complexité en formation : éducation, santé, travail social. Paris : Seli Arslan.

Saussez, F. et Allal, L. (2007). Réfléchir sur sa pratique : le rôle de l'autoévaluation? Mesure et évaluation en éducation, 30(1), 97-124.

Van Nieuwenhoven, C. et Colognesi, S. (2013). Une recherche collaborative autour des difficultés des maîtres de stage à accompagner leur stagiaire. Interraçoes, 9(27), 118-138. Repéré à

http://revistas.rcaap.pt/interaccoes/article/view/3405 